\title{
Estudo clínico e cardiorrespiratório em cadelas gestantes com parto normal ou submetidas à cesariana sob anestesia inalatória com sevofluorano
}

\author{
[Clinical and cardiorespiratory study in bitches under normal parturition or underwent to \\ cesarean section using inalatory anesthesia with sevoflurane] \\ D.T. Gabas $^{1 *}$, V.N.L.S. Oliva ${ }^{2}$, L.M. Matsuba ${ }^{1}$, S.H.V. Perri ${ }^{2}$ \\ ${ }^{1}$ Aluna de pós-graduação - FMVZ -UNESP - Botucatu, SP \\ ${ }^{2}$ Universidade Estadual Paulista Júlio de Mesquita Filho - UNESP - Araçatuba, SP
}

\begin{abstract}
RESUMO
Estudaram-se as alterações hemodinâmicas e respiratórias em cadelas, decorrentes do parto normal e da cesariana, utilizando-se sevofluorano como agente de manutenção anestésica. Foram acompanhados seis partos normais e seis cesarianas, sendo as últimas realizadas sob anestesia geral utilizando-se acepromazina, propofol e sevofluorano. Durante o parto normal, ao nascimento de cada filhote, as gestantes foram monitoradas (temperatura retal, pressão arterial não-invasiva, freqüências respiratória e cardíaca, tempo de reperfusão capilar e gasometria). Durante a cesariana foram avaliadas as mesmas características citadas para o parto normal, acrescentando-se a temperatura esofágica e a pressão arterial invasiva, ao longo de todo o período anestésico, além da qualidade da recuperação anestésica. Os valores das variáveis: freqüência cardíaca, pressão arterial, freqüência respiratória, tempo de reperfusão capilar e $\mathrm{o} \mathrm{pH}$ do sangue arterial no grupo submetido à cesariana foram menores que os das cadelas de partos normais, evidenciando a relativa depressão cardiorrespiratória produzida pelo procedimento anestésico. $\mathrm{O}$ protocolo anestésico empregado não comprometeu a viabilidade e a saúde das parturientes e dos filhotes e é seguro em cadelas gestantes, podendo ser utilizado nas operações cesarianas.
\end{abstract}

Palavras-chave: cadela, anestesia inalatória, sevofluorano, gestante, cesariana

\begin{abstract}
Haemodynamic and respiratory changes in 12 bitches, being six of natural birth and six caesarean using sevoflurane as an anaesthetic maintenance agent, were studied. The caesarean sections were performed using general anaesthesia, employing acepromazine maleate, propofol and sevoflurane. During natural birth, as each puppy was being delivered, the bitches were evaluated (rectal temperature, non-invasive arterial blood pressure respiratory and cardiac rate, capillary time reperfusion and blood gas values). During the caesarean, the same parameters mentioned for the natural birth were measured, in addition to the esophagus temperature and invasive arterial blood pressure throughout the anaesthetic period, besides the anaesthetic recovery quality. The bitches, submitted to the caesarean sections showed lower values of cardiac frequency, arterial pressure, respiratory frequency, time of capilar reperfusion and arterial blood $\mathrm{pH}$ when compared to the bitches which underwent natural birth, showing the cardiorespiratoy depression induced by the anaesthetic procedure. In spite of the anaesthetic depression, the anaesthetic protocol employed had no effect on the viability and health of the bitches and the puppies, and can be safely recommend for caesarean sections.
\end{abstract}

Keywords: bitch, inalatory anaesthesia, sevoflurane, pregnant, caesarean

Recebido em 30 de agosto de 2004

Aceito em 2 de março de 2006

*Endereço para correspondência (mailing address):

Rua Mem de Sá, 489 - 16065-090-Araçatuba, SP

E-mail: danigabas@hotmail.com 


\section{INTRODUÇÃO}

Em anestesiologia veterinária, o animal gestante deve ser considerado um paciente de alto risco, pois seu organismo está sujeito a uma série de alterações fisiológicas e, não raramente, patológicas decorrentes da gestação. Essas alterações comprometem a segurança do procedimento anestésico e devem ser criteriosamente analisadas, pois tanto a mãe quanto os fetos devem ser poupados, ao máximo, dos efeitos dos fármacos empregados.

Durante a gestação, a demanda metabólica na cadela aumenta, o que implica, diretamente, no aumento do débito cardíaco, do fluxo sangüíneo uterino, do consumo de oxigênio e da ventilação, com conseqüente diminuição da pressão parcial de dióxido de carbono do sangue arterial $\left(\mathrm{PaCO}_{2}\right)$ (Pascoe e Moon, 2001). No parto, a ventilação pode estar muito aumentada pelo efeito da dor, do medo e da ansiedade (Benson e Thurmon, 1987).

O aumento no débito cardíaco atinge cerca de 30 a $50 \%$ (Bicudo e Massone, 1990) e o volume sangüíneo também aumenta em cerca de $40 \%$, entretanto, as pressões sistólica e diastólica mantêm-se inalteradas (Thurmon et al., 1996).

Durante a gestação, as alterações fisiológicas alteram significativamente a função respiratória e diminuem suas reservas (Robertson e Moon, 2003). Pela ação progestacional, há aumento da sensibilidade do centro respiratório à pressão parcial de dióxido de carbono do sangue arterial $\left(\mathrm{PaCO}_{2}\right)$ (Gaido, 1997). Essa alteração é controlada com hiperventilação, aumento do volume minuto, resultando em hipocapnia e alcalose respiratória, compensada fisiologicamente com um aumento na excreção renal de bicarbonato (Massone, 2003).

É importante lembrar que durante a hipocapnia há aumento da afinidade da hemoglobina materna pelo oxigênio. Dessa forma, apesar da hiperventilação, pode haver hipóxia fetal (Gaido, 1997).

A alteração respiratória mais importante pela qual a futura mãe passa é a diminuição da capacidade residual funcional dos pulmões. Essa diminuição na reserva de oxigênio chega a 20 $25 \%$ no momento em que a paciente tem $20-35 \%$ de aumento do consumo de oxigênio, o que proporciona maior risco de hipóxia para a parturiente e, conseqüentemente, pode afetar o feto (Machado, 1997).

$\mathrm{Na}$ paciente gestante, a anestesia geral é a conduta mais indicada e segura, pois oferece ótimas condições de trabalho ao cirurgião, permitindo intubação traqueal, o que controla as vias aéreas da mãe, prevenindo a aspiração de conteúdo gástrico. Além disso, permite a manutenção materna sempre conectada à administração de oxigênio. Algumas desvantagens existem como, por exemplo, quando ocorre um plano anestésico superficial com liberação de catecolaminas pela mãe, diminuição da perfusão uterina e alterações severas da função cardiopulmonar (Steffey, 1996).

O sevofluorano, um anestésico halogenado recentemente introduzido na prática clínica, possui taxa de metabolização de 3,0\%, coeficiente de solubilidade gás/sangue de 0,68 e CAM em cães de 2,4\% (Natalini, 2001). Esse valor de CAM possibilita a classificação do sevofluorano como um anestésico de média potência e seu baixíssimo coeficiente de solubilidade resulta em duas de suas maiores vantagens que são a indução e a recuperação anestésicas bastante rápidas (Oliva et al., 2000).

Funkquist et al. (1997), ao estudarem dados referentes ao nascimento de 3.908 filhotes, obtiveram $71 \%$ de sobrevivência em cães nascidos de cesariana, sendo $26 \%$ natimortos e $3 \%$ dos filhotes morrendo nos primeiros minutos de vida em decorrência de traumatismos produzidos pela inabilidade materna. Luna et al. (2004) encontraram taxa de sobrevivência neonatal de $96 \%$ em cães nascidos de cesariana sob quatro diferentes protocolos anestésicos.

Há escassez de informações científicas a respeito dos efeitos materno e fetal da anestesia geral das cadelas gestantes e muitas são as situações em que a anestesia é necessária para a realização da operação cesariana eletiva ou de outras cirurgias no final da gestação. Dessa maneira, o trabalho teve por principal objetivo a avaliar as alterações cardiocirculatórias e respiratórias da cadela gestante, comparando o parto normal com o parto sob anestesia inalatória para a realização da operação cesariana. 


\section{MATERIAL E MÉTODOS}

Foram utilizadas oito cadelas, sem raça definida, com pesos entre 15 e $25 \mathrm{~kg}$. Como era necessário conhecer o período do ciclo estral de cada animal, na recepção deles realizou-se o exame de citologia vaginal, pelo menos uma vez por semana, a fim de acompanhar as mudanças de celularidade. Quando foram observados sangramento vaginal e edema vulvar (proestro), a citologia foi realizada três vezes por semana.

Após 10 dias do sangramento vaginal e do edema de vulva, na citologia vaginal observaram-se apenas células superficiais queratinizadas, com número reduzido de hemácias, característico do estro. A partir daí, as cadelas foram encaminhadas para cobriçao natural com cães. Quando elas não aceitavam a monta natural foi realizada a inseminação artificial com sêmen fresco.

Doze partos foram realizados sendo seis partos normais (GN) e seis cesarianas (GC), com a participação de oito cadelas diferentes, das quais quatro participaram dos dois protocolos. Nelas, o nascimento dos filhotes da primeira e da segunda gestação foi, respectivamente, de parto normal e de cesariana. As demais participaram de apenas um dos grupos, sendo duas de parto normal e duas de cesariana.

Para a determinação do momento correto de realização da cesariana eletiva e, conseqüentemente, da maturidade completa dos filhotes, foram analisados: tempo de gestação (60 a 63 dias decorridos da primeira cobertura), evolução radiográfica (visualização da pelve fetal aos 53 e aos 57 dias de gestação) (Feldman e Nelson, 1996) e diminuição da temperatura retal (Allen, 1995). A temperatura foi aferida duas vezes ao dia, uma semana antes da data prevista para o parto e quando a temperatura retal atingia $37,5^{\circ} \mathrm{C}$ ou ocorria a redução de $1{ }^{\circ} \mathrm{C}$ em relação à temperatura normal, considerando-se que o parto deveria ter início dentro das próximas 24 horas. Quando se constatava diminuição da temperatura passava-se a aferi-la no intervalo de três horas. Para a realização da cesariana eletiva considerou-se o momento em que a temperatura retal começava a se elevar.

Nos animais de parto normal, deixou-se que o parto evoluísse de maneira espontânea, realizando-se também os exames radiográficos, a monitoração da temperatura retal, a observação da eliminação do tampão mucoso e o tempo decorrido da cobrição. A evolução do trabalho de parto foi assistida pelos pesquisadores.

Os animais foram divididos em dois grupos para a realização do procedimento experimental. No $\mathrm{GN}$, após os primeiros sinais clínicos do começo do trabalho de parto, da elevação da temperatura, das primeiras contrações e da expulsão do tampão mucoso, avaliaram-se: temperatura retal ${ }^{1}$, (TR), freqüências cardíaca (FC) e respiratória ${ }^{2}$ (FR), pressões arteriais sistólica (PAS), média (PAM) e diastólica (PAD) obtidas pelo método não-invasivo por monitor de pressão ${ }^{3}$, e tempo de reperfusão capilar (TRC), por compressão digital na mucosa oral. Novas avaliações foram realizadas após cada nascimento.

Foram realizadas três coletas de sangue arterial $(1 \mathrm{ml})$ da mãe, a primeira antes do nascimento do primeiro filhote, a segunda após 60 minutos do primeiro nascimento e a terceira ao final do parto, para a realização de gasometria ${ }^{4}$.

No GC, a medicação pré-anestésica foi realizada com acepromazina ${ }^{5} \quad 0,2 \% \quad(0,05 \mathrm{mg} / \mathrm{kg}$, via intravenosa) e como agente indutor o propofol ${ }^{6}$ $(5 \mathrm{mg} / \mathrm{kg}$, via intravenosa).

A manutenção anestésica foi feita com sevofluorano $^{7}$ na concentração necessária para manter o segundo plano do terceiro estágio anestésico. Durante a manutenção anestésica realizou-se a cesariana conservativa e, independentemente do tempo necessário para tal procedimento cirúrgico, a anestesia foi mantida por 60 minutos contados a partir da estabilização do plano anestésico, mesmo que a cirurgia terminasse antes.

Decorridos 15 minutos da aplicação da medicação pré-anestésica foram mensuradas a TR, FC e FR e, na seqüência, realizaram-se a

\footnotetext{
${ }^{1}$ Termômetro clínico -BD

${ }^{2}$ Estetoscópio- Littmann

${ }^{3}$ Monitor de pressão arterial - Dixtal - DX 2710

${ }^{4}$ Aparelho de gasometria-Drake AGS 12

${ }^{5}$ Acepran $0,2 \%$ - Univet S.A. Industria Veterinária

${ }^{6}$ Propofol - Cristália Produtos Químicos e Farmacêuticos Ltda.

${ }^{7}$ Sevocris - Cristália Produtos Químicos e farmacêuticos Ltda.
} 
indução anestésica (bolus), a intubação orotraqueal e a conexão a um sistema circular de anestesia.

A veia cefálica foi canulada com cateter $20 \mathrm{~g}$ e mantida com fluidoterapia, Ringer com lactato $10 \mathrm{ml} / \mathrm{kg} / \mathrm{h}$. A manutenção anestésica foi realizada com sevofluorano em fluxo diluente de oxigênio (1 a 1,5/min), em sistema circular de anestesia. Decorridos 15 minutos do início da vaporização do agente, com o plano anestésico estabilizado, foram mensuradas, no intervalo de cinco minutos as características: TR, temperatura esofágica $^{8}, \mathrm{FR}$, tensão de dióxido de carbono no final da expiração $\left(\mathrm{ETCO}_{2}\right)^{9}, \mathrm{FC}, \mathrm{TRC}, \mathrm{PAS}$, PAM e PAD pelo método não-invasivo, e PAM pelo método invasivo (cateterização transcutânea da artéria femoral, com cateter $20 \mathrm{~g}$ e conexão a manômetro aneróide). ${ }^{10}$

No GC, a primeira coleta de sangue para gasometria ${ }^{4}$ foi realizada com seringa de insulina antes da aplicação de qualquer fármaco. Após a indução, foi realizada a cateterização transcutânea da artéria femoral, com cateter $20 \mathrm{~g}$ e foi acoplada uma torneira de três vias, onde foi coletada a segunda amostra após 30 minutos da manutenção anestésica e a terceira amostra ao final dos 60 minutos.

Para a realização da análise estatística foram considerados os momentos basais (MI) e as médias de todos os momentos (MF) correspondentes a cada nascimento no GN e no GC.

\section{RESULTADOS E DISCUSSÃO}

Durante o parto normal, os valores de freqüência cardíaca da parturiente mantiveram-se acima do considerado normal para a espécie. Este resultado é esperado, uma vez que, em razão do aumento da demanda metabólica na cadela gestante, há aumento do débito cardíaco e da freqüência cardíaca (Thurmon et al., 1996). Além disso, é bastante presumível que o trabalho de parto, em si, exija maior esforço físico, resultando em aumento de trabalho cardíaco. A anestesia reduziu de maneira significativa a freqüência cardíaca. Este resultado difere dos

\footnotetext{
${ }^{8}$ Monitor de sinais vitais 9505 - K-TAKAOKA

${ }^{9}$ Aparelho Oxigel- modelo 800

${ }^{10}$ Marca Heidji
}

relatados por Mutoh et al.(1997) e Oliva et al. (2000), que observaram aumento da FC em relação aos valores basais, com o uso de sevofluorano em cães (Fig. 1).

Durante o parto, a ventilação pode estar muito aumentada pelo efeito da dor e das contrações (Benson e Thurmon, 1987), justificando, assim, os valores médios elevados da FR nas cadelas durante o trabalho de parto, ainda que este aumento não tenha sido estatisticamente significativo. Essa taquipnéia pode ser facilmente explicada pelo esforço físico necessário para o parto normal.

Nas cadelas submetidas à cesariana, os valores daquela variável foram significativamente inferiores aos observados no grupo GN. Isso se deveu, certamente, ao encaminhamento dos animais para a cesariana antes do início das contrações e, portanto, sem o efeito de dor e do maior trabalho muscular. Além disso, esse efeito pode ser atribuído aos fármacos utilizados no procedimento anestésico, como observado por Mutoh et al. (1997), ainda que esses autores tenham constatado depressão respiratória significativa em cães apenas quando se usou concentração a partir de dois CAM de sevofluorano (Fig. 2).

Thurmon et al. (1996) afirmaram que, apesar do aumento de débito cardíaco e do volume sangüíneo ocorrer na gestação, as pressões sistólica e diastólica permanecem inalteradas. Neste trabalho, observou-se que os valores iniciais da PAS foram mais elevados nas cadelas do GN do que naquelas que, ainda não estando em trabalho de parto, foram encaminhadas ao procedimento cirúrgico, levando-se à suposição de que o aumento da demanda metabólica requerida pelo parto seja o responsável pela elevação.

Os valores da PAM e da PAD iniciais nas cadelas do GC foram maiores que os valores médios obtidos durante a manutenção anestésica. Massone (2003) descreveu que o halotano deve ser evitado em pacientes de alto risco devido à intensa hipotensão. O sevofluorano, apesar de também causar diminuição da pressão arterial, mantém valores aceitáveis desta, o que pode 
indicá-lo com um agente mais seguro para a utilização em animais com gestação a termo. Durante o trabalho de parto normal, as PAM e PAD mantiveram-se constantes ao longo de toda a observação, o que pode ser explicado pela ausência de fármacos nesses pacientes, ao contrário do que ocorreu no grupo submetido à cesariana.

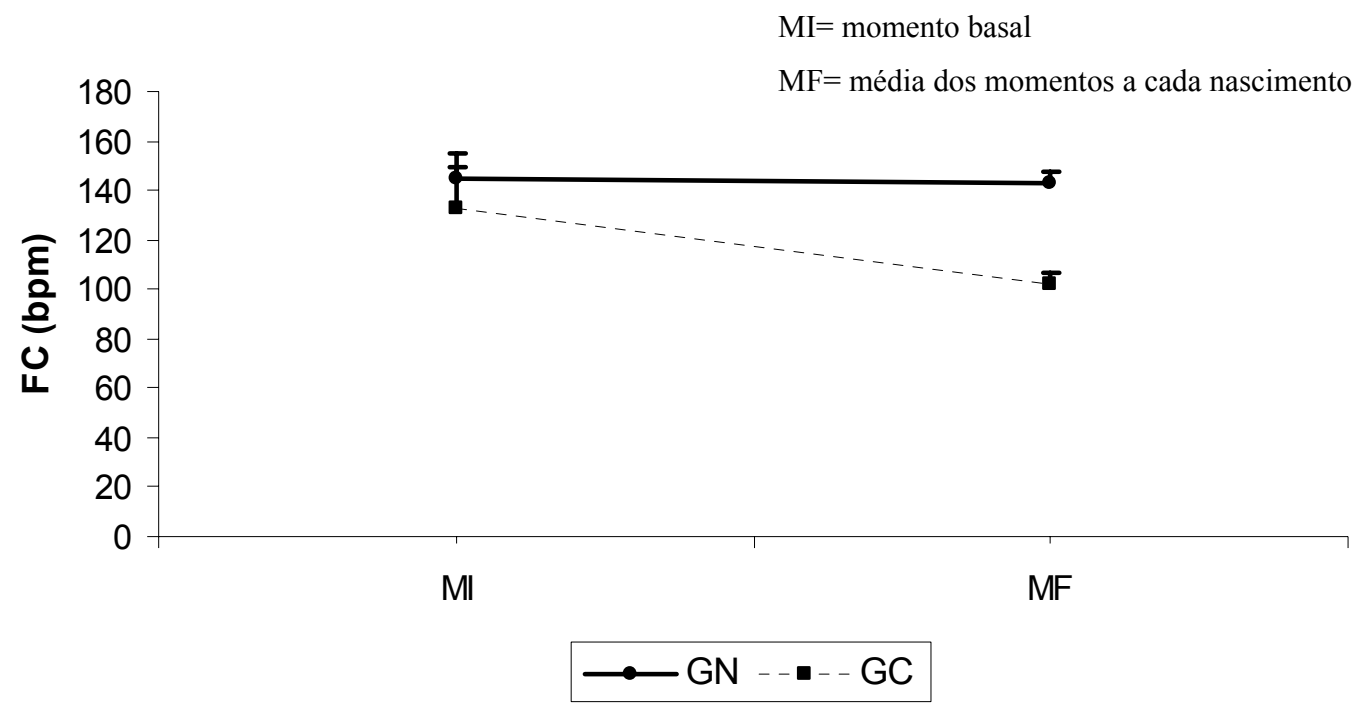

Figura 1. Média e erro-padrão da média da freqüência cardíaca (FC), em bpm, em cadelas de parto normal (GN), ou submetidas à cesariana (GC), anestesiadas com sevofluorano e pré-tratadas com acepromazina $(0,05 \mathrm{mg} / \mathrm{kg})$ e propofol $(5 \mathrm{mg} / \mathrm{kg})$.

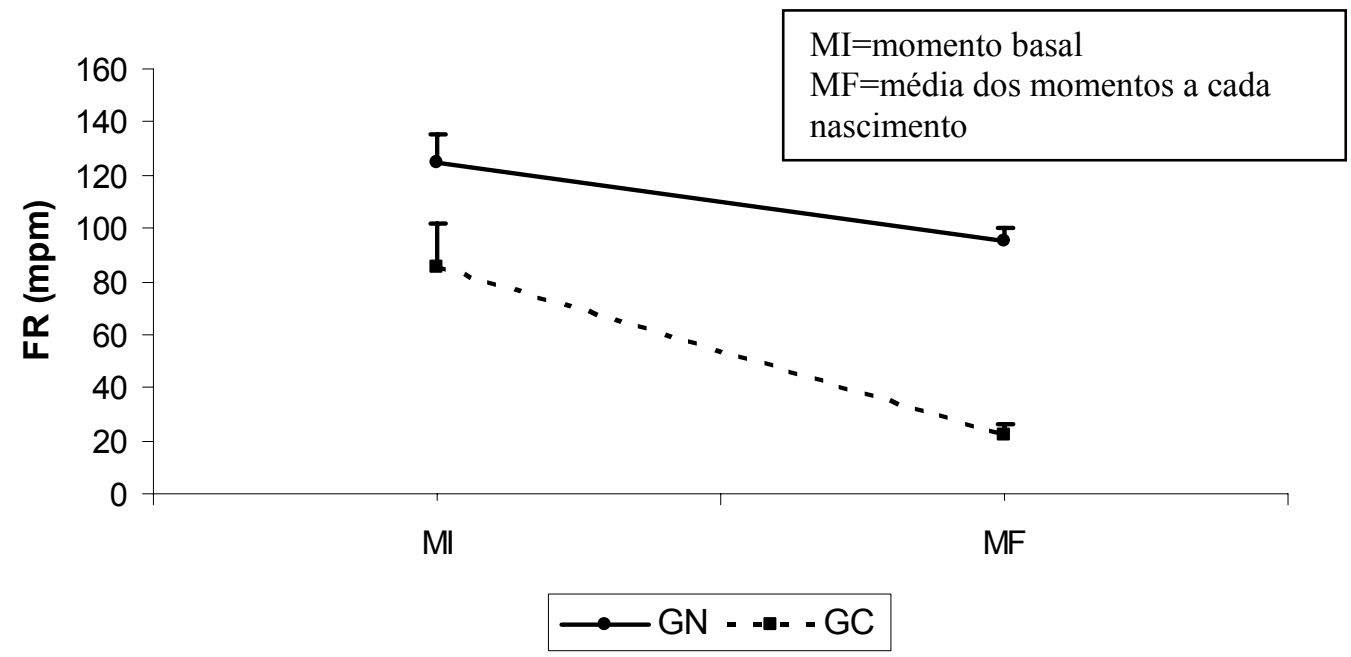

Figura 2. Média e erro-padrão da média da freqüência respiratória (FR), em mpm, em cadelas de parto normal (GN), ou submetidas à cesariana (GC), anestesiadas com sevofluorano e pré-tratadas com acepromazina $(0,05 \mathrm{mg} / \mathrm{kg})$ e propofol $(5 \mathrm{mg} / \mathrm{kg})$. 
No grupo GN não foi observada alteração do tempo de reperfusão capilar, provavelmente devido à manutenção da pressão arterial em valores normais para a espécie. Porém, nos animais do grupo $\mathrm{GC}$, ocorreu aumento significativo dessa característica durante a manutenção anestésica, de maneira inversamente proporcional à diminuição das pressões arteriais média e diastólica.

A temperatura retal diminui gradativamente ao longo da manutenção anestésica realizada durante a cesariana do GC. Isso deveu-se, provavelmente, ao efeito dos fármacos utilizados como protocolo anestésico. Eles deprimem o mecanismo de termorregulação, reduzem o metabolismo basal e aumentam a perda de calor por meio da vasodilatação (Yazbek, 2002). A temperatura ambiente e a exposição da cavidade abdominal ao meio externo, certamente também contribuíram para essa diminuição. A temperatura retal dos animais do GN manteve-se estável ao longo de todo o trabalho de parto, provavelmente porque nesse grupo não foram utilizados fármacos e não foi realizado o procedimento cirúrgico.

Apesar de os valores de $\mathrm{pH}$ do sangue arterial no GC ter diminuído de maneira significativa ao longo do procedimento anestésico e de ter-se constatado redução da FR, os valores da pressão parcial de dióxido de carbono no sangue arterial $\left(\mathrm{PaCO}_{2}\right)$ e de dióxido de carbono total no sangue arterial $\left(\mathrm{CO}_{2} \mathrm{~T}\right)$ não aumentaram, diferente do que seria esperado. Durante a gestação, as alterações fisiológicas alteram significativamente a função respiratória, com aumento da sensibilidade do centro respiratório à pressão parcial de dióxido de carbono do sangue arterial (Gaido, 1997). Para controlar essas alterações, há hiperventilação com aumento do volume/minuto resultando em hipocapnia e alcalose respiratória, com excreção renal compensatória de bicarbonato (Massone, 2003). Nas cadelas submetidas ao parto normal ocorreu hiperventilação, com hipocapnia, confirmando o relato desses autores.

Houve significativa elevação da pressão parcial de oxigênio $\left(\mathrm{PaO}_{2}\right)$ no sangue arterial e da saturação de oxigênio $\left(\mathrm{SatO}_{2}\right)$ nos momentos de manutenção anestésica do grupo $\mathrm{GC}$, o que pode ser facilmente explicado pela suplementação de oxigênio utilizada durante $\mathrm{o}$ procedimento anestésico, o que não ocorreu nas cadelas submetidas ao parto normal, nas quais os valores daquelas variáveis mantiveram-se estáveis.

Não houve diferença na concentração de bicarbonato $\left(\mathrm{HCO}_{3}{ }^{-}\right)$no sangue arterial entre os momentos de cada grupo nem entre os grupos, visto que os valores médios de $\mathrm{pH}$ estavam dentro de valores normais para a espécie.

No GN não houve diferença significativa entre os momentos nem entre os grupos em relação ao excesso de bases (BE) no sangue arterial, provavelmente por não ter ocorrido alteração significativa do $\mathrm{pH}$ neste grupo. No GC observou-se excesso de base tentando compensar a diminuição do $\mathrm{pH}$ ocorrida no período de manutenção.

A taxa de sobrevivência neonatal foi de $97,1 \%$ no grupo de animais nascidos de parto normal enquanto que, nos nascimentos ocorridos através de cesariana esta taxa foi de 91,4\%. Estes resultados assemelham-se aos de Funkquist et al. (1997) e Luna et al. (2004), e referem-se tanto ao número de animais natimortos quanto àqueles mortos nas primeiras 24 horas decorridas do nascimento. Entre os 33 filhotes nascidos de parto normal e 32 nascidos de cesariana, ocorreu um caso de natimorto em cada grupo.

\section{CONCLUSÕES}

A anestesia geral com acepromazina, propofol e sevofluorano provoca relativa depressão cardíaca e acidemia nas cadelas gestantes submetidas a cesariana. Apesar dessas alterações, o protocolo anestésico utilizado mostrou-se seguro para a mãe e para os fetos e constitui uma boa opção para a anestesia geral de cadelas gestantes, ao produzir taxa de mortalidade neonatal muito semelhante à que ocorre no parto normal.

\section{REFERÊNCIAS BIBLIOGRÁFICAS}

ALLEN, E.W. Fertilidade e obstetrícia no cão. São Paulo: Varela, 1995. p.61-76.

BENSON, G.J.; THURMON, J.C. Clinical anesthesia in various animal species and condictions: special anesthetic considerations for 
cesarean section. In: SHORT, C. E. Principles and practice of veterinary anesthesia. Baltimore: Williams \& Wilkins, 1987. cap.13, p.337-348.

BICUDO, S.D.; MASSONE, F. Modificações fisiológicas durante a gestação e suas implicações na anestesia em cadelas prenhes. Cães \& Gatos, v.31, p.4-7, 1990.

DODMAN, N.H. Anaesthesia for caesarean section in the dog and cat: a review. J. Small Anim. Pract., v.20, p.449-460, 1979.

FELDMAN, E.C.; NELSON, R.W. Breeding, pregnancy, and parturion. In: . Canine and feline endocrinology and reproduction. 2.ed. Philadelfia: W.B. Saunders, 1996. cap.18, p.559. (34)

FUNKQUIST, P.M.E.: NYMAN, G.C.; LÓFGREEN, A.M.J. et al. Use of propofolisoflurane as an anesthetic regimen for cesarean section in dogs. J. Am. Vet. Med. Assoc., v.211, p.313-317,1997.

GAIDO, S.R. A gestação e a anestesia. In: ENCONTRO DE ANESTESIOLOGIA VETERINÁRIA, 3., 1997, Araçatuba. Anais... Araçatuba: Colégio Brasileiro de Cirurgia e Anestesiologia Veterinária, 1997. p.10-16.

GREENE, S.A. Anesthetic considerations for surgery of the reproductive system. Semin. Vet. Med. Surg. (Small Anim.), v.10, p.2-7, 1995.

LUNA, S.P.L.; CASSU, R.N.; CATRO, G.B. et al. Effects of four anaesthetic protocols on the neurological and cardiorespiratory variables of puppies born by caesarean section. Vet. Rec., v.154, p.387-389, 2004.

MACHADO, C.E.G. Anestesia na paciente gestante: uma abordagem em pequenos animais. In: ENCONTRO DE ANESTESIOLOGIA VETERINÁRIA, 3., 1997, Araçatuba. Anais... Araçatuba: Colégio Brasileiro de Cirurgia e Anestesiologia Veterinária, 1997. p.17-34.

MASSONE, F. Anestesiologia veterinária: farmacologia e técnica. 4.ed. Rio de Janeiro:
Guanabara Koogan, 2003. p.1-17, 195-198, 205210.

MUTOH, T.; NISHIMURA, R.; KIM, H. Y. et al. Cardiopulmonary effects of sevoflurane, compared with halothane, enflurane, and isolfurane, in dogs. Am. J. Vet. Res., v.58, p.885890, 1997.

NATALINI, C.C. Sevoflurane, Desflurane, and Xenon new inhaled anesthetics in veterinary medicine. Ciên. Rural, v.31, p.177-183, 2001.

NUNES, N. Monitoração da anestesia. FANTONI, D.T.; CORTOPASSI, S.R.G. Anestesia em cães e gatos. São Paulo: Roca, 2002. p.64-81.

OLIVA, V.N.L.S.; MASSONE, F.; TEIXEIRA NETO, F.J. et al. Avaliação cardiocirculatória do sevofluorano como agente de manutenção anestésica em cães, em diferentes concentrações de óxido nitroso. Arq. Bras. Med. Vet. Zootec., v.52, p.130-137, 2000.

PASCOE, P.J.; MOON, P.F. Periparturient and neonatal anesthesia. Vet. Clin. N. Am.: Small Anim. Pract., v.31, p.315-340, 2001.

ROBERTSON, S.A.; MOON, P.F. Anesthetic management for cesarean section in bitches. Vet. Med., v.98, p.675-696, 2003.

STEFFEY, E.P. Inhalation anesthetic. In: THURMON, J.C.; TRANQUILLI, W.J.; BENSON, G.J. (Eds.). Lumb \& Jones' veterinary anesthesia. 3.ed. Philadelfia: Lippincott Willians \& Wilkins, 1996. p.297-323.

THURMON, J.C.; TRANQUILLI, W.J.; BENSON, G.J. Anaesthesia for special patients: cesarean section patients. In: Lumb \& Jones' veterinary anaesthesia. 3.ed. Philadelfia: Lippincott Williams \& Wilkins, 1996. p.818828.

YAZBEK, K.V.B. Hipotermia. In: FANTONI, D.T.; CORTOPASSI, S.R.G. Anestesia em cães e gatos. São Paulo: Roca, 2002. p.372-378. 